\title{
Rietveld Neutron Powder Profile Analysis and Electrical Conductivity of the Fast Silver-Ion Conductor (LaO) AgS
}

\author{
Dirk Wilmer \\ University of Münster, Institute of Physical Chemistry, Münster Germany \\ Bernhardt J. Wuensch \\ Department of Materials Science and Engineering, MIT, Cambridge, MA
}

\author{
J. D. Jorgensen
}

IPNS, Argonne National Laboratory, Argonne, IL

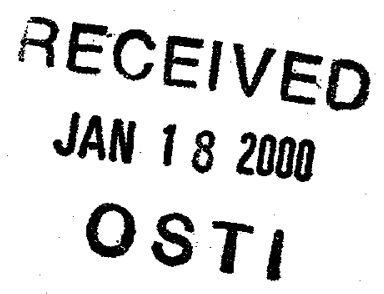

To be submitted to the Fall Material Research Society Conference, Boston, MA, November 29 - December 3, 1999

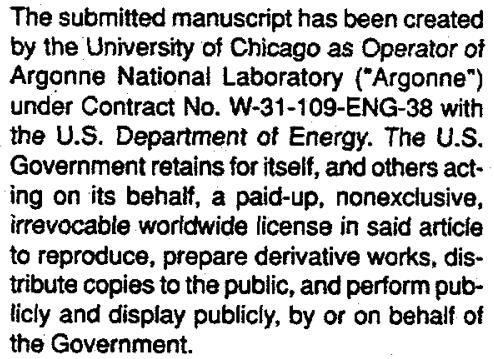

This work was supported by the U.S. Department of Energy, Office of Basic Energy Sciences Division of Materials Sciences, under contract \#W-31-109-ENG-38. 


\section{DISCLAIMER}

This report was prepared as an account of work sponsored by an agency of the United States Government. Neither the United States Government nor any agency thereof, nor any of their employees, make any warranty, express or implied, or assumes any legal liability or responsibility for the accuracy, completeness, or usefuiness of any information, apparatus, product, or process disclosed, or represents that its use would not infringe privately owned rights. Reference herein to any specific commercial product, process, or service by trade name, trademark, manufacturer, or otherwise does not necessarily constitute or imply its endorsement, recommendation, or favoring by the United States Government or any agency thereof. The views and opinions of authors expressed herein do not necessarily state or reflect those of the United States Government or any agency thereof. 


\section{DISCLAIMER}

Portions of this document may be illegible in electronic image products. Images are produced from the best available original document. 


\title{
RIETVELD NEUTRON POWDER PROFILE ANALYSIS AND ELECTRICAL CONDUCTIVITY OF THE FAST SILVER-ION CONDUCTOR (LaO)AgS
}

\author{
DIRK WILMER* , BERNHARDT J. WUENSCH**, JAMES D. JORGENSEN \\ *University of Münster, Institute of Physical Chemistry, Münster, Germany \\ ** Department of Materials Science and Engineering, MIT, Cambridge, MA \\ $\dagger$ IPNS, Argonne National Laboratory, Argonne, IL
}

\begin{abstract}
Lanthanum silver oxysulfide, (LaO)AgS, exhibits a predominantly ionic conductivity of $10^{-3}$ to $10^{-1} \mathrm{~S} / \mathrm{cm}$ between $300 \mathrm{~K}$ and $770 \mathrm{~K}$. The tetragonal structure consists of alternating $(\mathrm{LaO})$ and (AgS) sheets, their sequence being O-La-S-Ag-S-La-O. The structure suggests that ionic transport arises from migration of silver ions within the AgS layers analogous to sodium ion transport in Na- $\beta$-alumina.

Neutron powder diffraction data measured at five temperatures between $300 \mathrm{~K}$ and $770 \mathrm{~K}$ are analysed using the Rietveld method to determine the distribution and thermal vibration parameters of the mobile silver ions. The structural investigation is accompanied by measurements of the total conductivity in the same temperature range in order to resolve severe discrepancies in the literature data.
\end{abstract}

\section{INTRODUCTION}

In 1980, Palazzi et al. [1] reported a new crystalline silver fast-ion conductor, (LaO)AgS which they obtained by solid state reaction of a mixture of $\mathrm{La}_{2} \mathrm{O}_{2} \mathrm{~S}$ and $\mathrm{Ag}_{2} \mathrm{~S}$ at $580^{\circ} \mathrm{C}$ to $640^{\circ} \mathrm{C}$ in the presence of a small amount of iodine. They performed conductivity measurements (ac impedance, $\mathrm{dc}$ using blocking electrodes) and concluded that the conductivity is mainly ionic, with an activation energy of $(0.195 \pm 0.05) \mathrm{eV}$. The structure was solved by the same group [2]: the tetragonal cell (S.G. P4/nmm, with $a=4.050 \AA$ and $c=9.039 \AA$ ) contains two formula units.

The structure of $(\mathrm{LaO}) \mathrm{AgS}$ consists of eight-coordinated La ions (position $2 \mathrm{c} \frac{1}{4} \frac{1}{4} z$, with $z=$ $0.1356)$ between a sheet of oxygen ions $\left(2 \mathrm{a} \frac{3}{4} \frac{1}{4} 0\right)$ and a sheet of sulfur ions (2c $\frac{1}{4} \frac{1}{4} z$, with $z=$ $0.6929)$ linked to form a layer of composition $\mathrm{LaO}_{2} \mathrm{~S}$. The silver ions $\left(2 \mathrm{~b} \frac{3}{4} \frac{1}{4} \frac{1}{2}\right)$ are situated in tetrahedral coordination between a pair of sulfur layers. The $\mathrm{AgS}_{4}$ tetrahedra share four of their six edges to form a second type of polyhedral layer of composition AgS. Each sheet of $\mathrm{S}$ ions is common :o a LaO $\mathrm{La}_{2} \mathrm{~S}$ layer and an $\mathrm{AgS}$ layer; the $\mathrm{O}$ layer is shared between a pair of $\mathrm{LaO}_{2} \mathrm{~S}$ layers. The sequence of layers along $c$ is thus O-La-S-Ag-S-La-O. The layered nature of the ( $\mathrm{LaO}) \mathrm{AgS}$ structure, with edge-sharing tetrahedral layers separated by two intervening $\mathrm{LaO}_{2} \mathrm{~S}$ slabs, suggests a two-dimensional enhancement of silver mobility within (001).

A few years later, Ishikawa et al. [3] re-examined the electrical properties of (LaO)AgS. They reported difficulties in obtaining pure samples when using Palazzi's method and used a sulfur atmosphere instead of iodine. The total conductivities they determined showed an activation energy of $0.30 \mathrm{eV}$ and, at about $100^{\circ} \mathrm{C}$, were more than two orders of magnitude lower than the values previously reported [1]. In addition, both their ionic and total conductivities exhibited a stepwise increase at about $180^{\circ} \mathrm{C}$ which was not explained. (LaO)AgS was classified as an $n$-type mixed conductor.

In 1995, the same group published new results for (LaO)AgS [4], now giving an activation energy for the ionic conductivity of $0.107 \mathrm{eV}$. Although the data in their Arrhenius-type plots of ionic and total conductivities are very close together, their results from two different measurements of the silver transference number yield values of 0.066 and 0.48 , respectively. Moreover, an excess of $\mathrm{La}_{2} \mathrm{O}_{2} \mathrm{~S}$ had to be added in order to suppress the stepwise increase of conductivities at around $180^{\circ} \mathrm{C}$.

The main goal of the present study is to examine the structural properties which determine the silver ion transport mechanism in ( $\mathrm{LaO}) \mathrm{AgS}$. We, therefore, have performed neutron time-offlight diffraction experiments on ( $\mathrm{LaO}) \mathrm{AgS}$ at different temperatures in order to use changes in site 
occupancies and thermal vibration amplitudes to gain information on preferred conduction paths and their dimensionality. Since the published conductivity data are not consistent with each other, we have included a preliminary report on conductivity measurements that we have performed.

\section{EXPERIMENTAL}

\section{Sample preparation}

Powder samples of ( $\mathrm{LaO}) \mathrm{AgS}$ were prepared using Palazzi's method [1]. Stochiometric mixtures of dried $\mathrm{La}_{2} \mathrm{O}_{2} \mathrm{~S}$ (Cerac Inc.) and $\mathrm{Ag}_{2} \mathrm{~S}$ (ALFA) were heated with a trace of iodine to $620^{\circ} \mathrm{C}$ in evacuated and sealed Vycor ampoules. Mean heating times were about 100 hours. The bright green product was checked for purity by powder X-ray diffraction. Contrary to other reports $[3,4]$ we encountered no severe difficulties in obtaining the product as a single phase.

\section{Total conductivity}

Powder samples were uniaxially pressed into cylindrical disks at $4 \mathrm{kpsi}$, then isostatically at $40 \mathrm{kpsi}$, yielding about $80 \%$ of the theoretical density. Conditions under which the samples may be sintered are restricted as the material decomposes at temperatures above ca. $1000 \mathrm{~K}$. The flat faces of the samples were covered with silver electrodes by thermal evaporation. Two-probe measurements were performed with a Solartron 1260 . Samples were kept in a flow of dry nitrogen to prevent oxidation at elevated temperatures. The electrical tube furnace was controlled by a Eurotherm 818.

\section{Neutron diffraction}

Time-of-flight neutron diffraction data were collected at five temperatures between $300 \mathrm{~K}$ and $773 \mathrm{~K}$ with the Special Environment Powder Diffractometer (SEPD) at the Intense Pulsed Neutron Source (Argonne National Laboratory) [5]. For the measurements at elevated temperatures, the powder was placed in a thin-walled cylindrical vanadium container; the room temperature measurement was performed with a pressed pellet without container. Diffraction profiles were recorded at three different values for $2 \theta\left(145^{\circ}, 90^{\circ}, 60^{\circ}\right)$ and were employed as discrete coupled data sets in the analysis.

\section{Rietveld analysis}

Simultaneous refinements of the three powder profiles were performed with the GSAS suite of programs [6]. A few limited regions of the recorded profiles at elevated temperatures contained diffraction maxima from the sample holder and furnace and were excluded from the refinements.

A total of 34 parameters were required for three powder profiles in the model: a scale factor, five background parameters and a time-of-flight zero-point offset for each powder profile; two lattice constants, two positional parameters and 8 anisotropic thermal parameters to describe the structure; a single parameter was used to describe absorption for all three powder profiles. Final weighted residuals $\left(R_{w p}\right)$ for the refinements ranged from $6.1 \%$ to $10.6 \%$, the corresponding expected values $\left(R_{e}\right)$ between $4.1 \%$ and $6.2 \%$.

\section{RESULTS AND DISCUSSION}

\section{Total conductivity}

Figure 1 shows the total conductivities of $(\mathrm{LaO}) \mathrm{AgS}$ in a logarithmic plot versus reciprocal temperature. The data were obtained using three different samples; they provide values for the conductivity that agree within reasonable limites. The activation energy is $0.22 \mathrm{eV}$, slightly higher than Pallazi's result of $0.195 \mathrm{eV}$. Although the absolute values of the conductivity are lower than those reported by Palazzi et al. [1], the general trend is more or less the same. It is worth noting 
that the remarkable conductivity increase at around $180^{\circ} \mathrm{C}$ found by Ishikawa et al. [4] could not be detected. Generally speaking, our results confirm the findings of Palazzi's experiments.

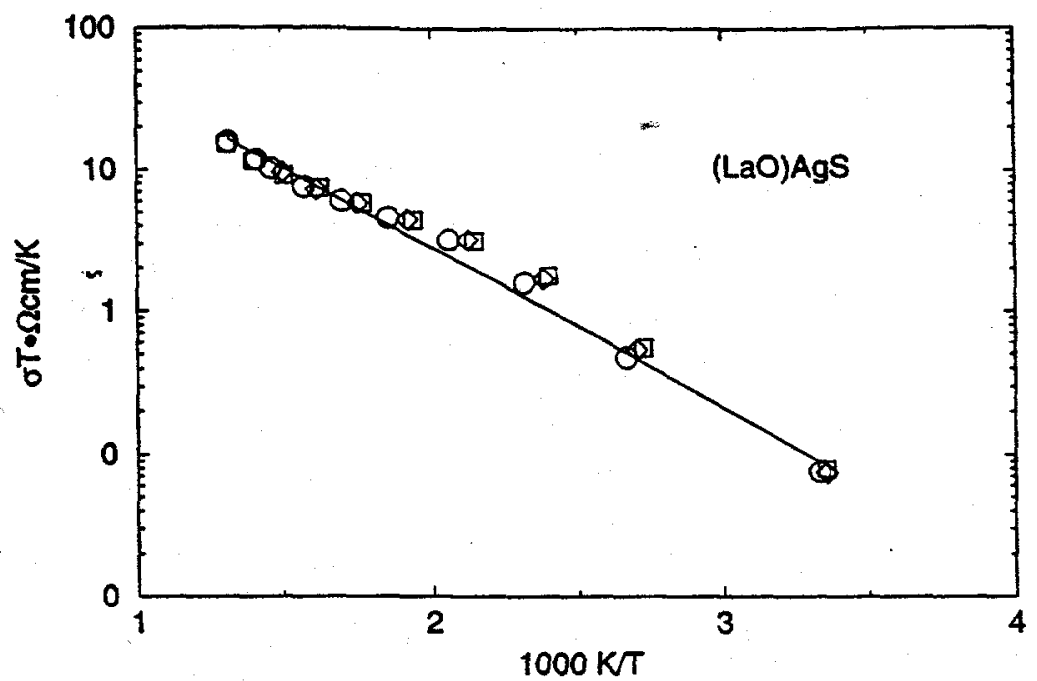

Figure 1: Arrhenius plot for the total conductivities of $(\mathrm{LaO}) \mathrm{AgS} . E_{a}=0.22 \mathrm{eV}$. Values shown are from three different samples.

\section{Neutron diffraction}

In Fig. 2 we present the temperature-dependent lattice parameters, which, as expected, show a linear trend. The linear thermal expansion coefficients are $(1.61 \pm 0.07) \cdot 10^{-5} \mathrm{~K}^{-1}$ and $(2.78 \pm$ $0.03) \cdot 10^{-5} \mathrm{~K}^{-1}$ for $a$ and $c$, respectively. The refined values of the $z$ coordinate for $\mathrm{La}$ and $\mathrm{S}$ are
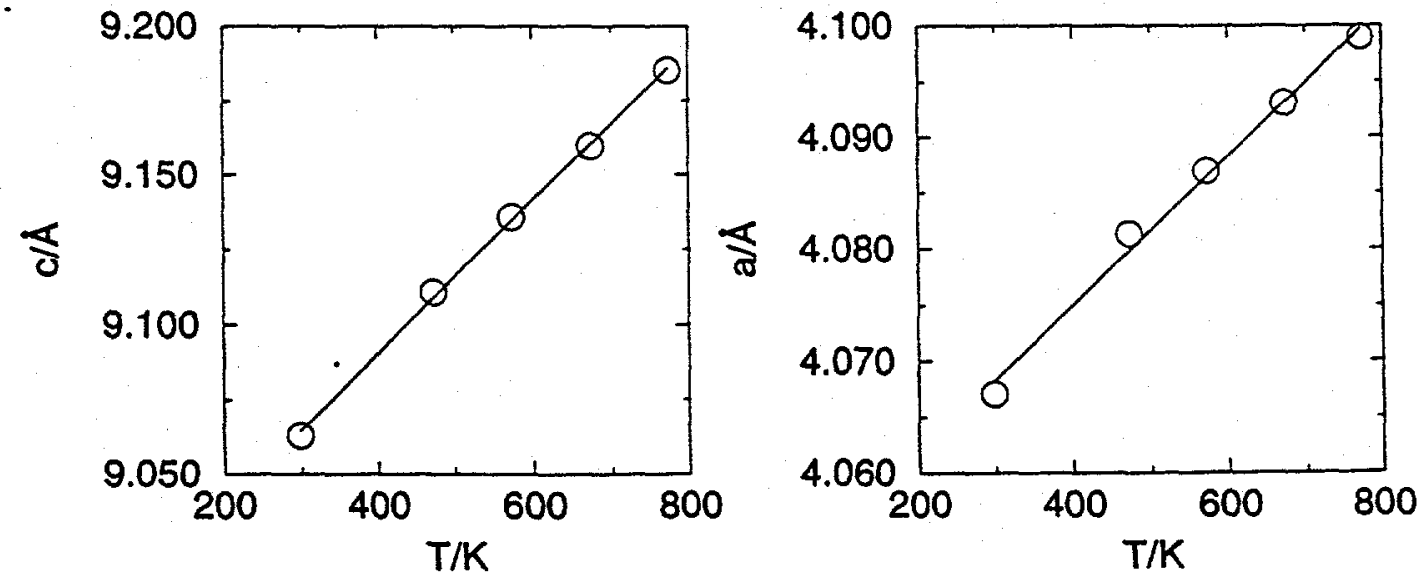

Figure 2: Lattice parameters of $(\mathrm{LaO}) \mathrm{AgS}$ as a function of temperature.

given in Table 1. Anisotropic temperature factor coefficients as defined in

$$
T=\exp \left[-2 \pi^{2}\left(u_{11} h^{2} a^{* 2}+\ldots+2 u_{12} h k a^{*} b^{*}+\ldots\right)\right]
$$

are presented in Table 2 . Due to symmetry, all $u_{i j}$ with $i \neq j$ are zero, and $u_{11}=u_{22}$. Not surprisingly, the $u_{i i}$ for the mobile silver ions are considerably higher than for all other ions in the system. In addition, $u_{11}(\mathrm{Ag})>u_{33}(\mathrm{Ag})$, supporting the view that silver ions reside in a potential that is shallower in a direction normal to $c$ and that they move preferentially in the $a-b$-plane. 


\begin{tabular}{llllll}
\hline$T / \mathrm{K}$ & 298 & 473 & 573 & 673 & 773 \\
\hline \hline$z(\mathrm{La})$ & $0.13612(13)$ & $0.13717(26)$ & $0.13680(30)$ & $0.13610(30)$ & $0.13477(31)$ \\
$z(\mathrm{~S})$ & $0.69432(37)$ & $0.69842(56)$ & $0.69898(59)$ & $0.69885(61)$ & $0.69772(66)$ \\
\hline
\end{tabular}

Table 1: Refined values of the positional parameters.

\begin{tabular}{lllllllll}
$T T / K$ & $u_{11}(\mathrm{Ag})$ & $u_{33}(\mathrm{Ag})$ & $u_{11}(\mathrm{La})$ & $u_{33}(\mathrm{La})$ & $u_{11}(\mathrm{O})$ & $u_{33}(\mathrm{O})$ & $u_{11}(\mathrm{~S})$ & $u_{33}(\mathrm{~S})$ \\
\hline \hline 298 & $3.67(8)$ & $2.96(12)$ & $0.63(3)$ & $1.38(7)$ & $0.58(5)$ & $1.28(9)$ & $0.86(10)$ & $1.66(19)$ \\
473 & $5.42(19)$ & $3.09(27)$ & $0.63(7)$ & $2.47(17)$ & $0.34(9)$ & $1.82(18)$ & $0.65(19)$ & $0.83(35)$ \\
573 & $7.94(26)$ & $3.08(30)$ & $0.59(7)$ & $3.21(19)$ & $0.80(9)$ & $1.60(19)$ & $1.59(22)$ & $0.88(37)$ \\
673 & $8.24(27)$ & $4.07(34)$ & $0.71(7)$ & $3.61(21)$ & $1.10(11)$ & $1.14(19)$ & $1.18(23)$ & $1.76(45)$ \\
773 & $8.09(26)$ & $6.22(42)$ & $0.92(7)$ & $3.77(22)$ & $1.40(11)$ & $1.19(20)$ & $1.51(25)$ & $2.41(53)$ \\
\hline
\end{tabular}

Table 2: Anisotropic temperature factor coefficients (in units of $100 \AA^{2}$ ) with estimated standard deviations in parentheses.

For the immobile ions $(\mathrm{La}, \mathrm{O}, \mathrm{S})$, the relation of the anisotropic temperature factors is reversed: they all show $u_{11}<u_{33}$. A tentative explanation is that when silver ions move in the $a-b$-plane, the other ions have to give way by moving along the $c$ axis.

The estimated standard deviations that are given are those based on counting statistics. One must, however, keep in mind that for the present case of a strongly absorbing sample, the refined values of the temperature factor coefficients are strongly influenced by the absorption correction. We, therefore, restrict ourselves to a more qualitative analysis.
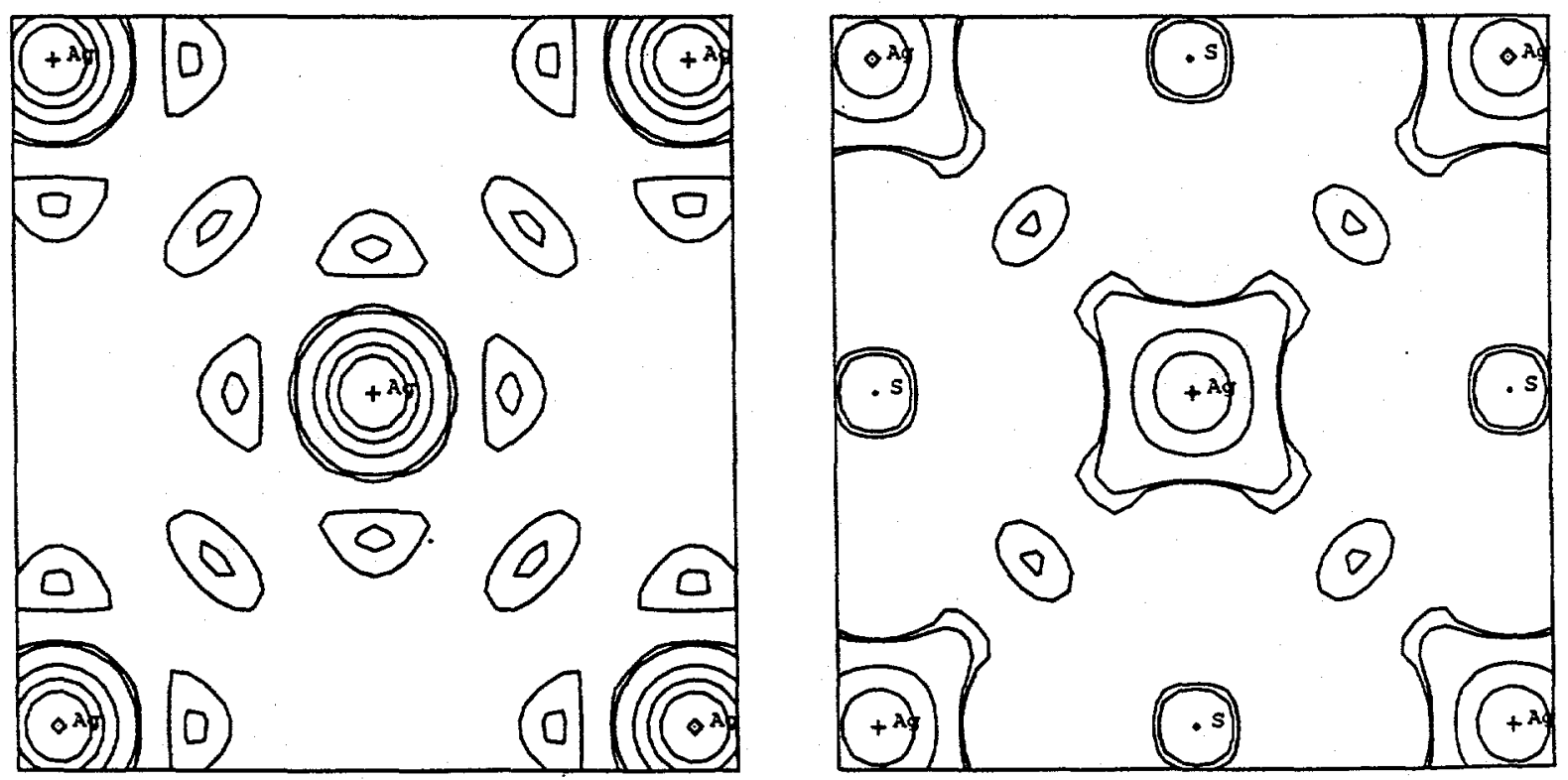

Figure 3: Fourier section $\left(F_{o b s}\right)$ at $z=\frac{1}{2}$ for $(\mathrm{LaO}) \mathrm{AgS}$ at $298 \mathrm{~K}$ (left) and $473 \mathrm{~K}$ (right). The silver ion at the center of the diagram is located at position $\frac{3}{4} \frac{1}{4} \frac{1}{2}$. Contour values are $0.10,0.20,1.00$, $2.00,3.00$ (left) and $0.12,0.17,1.00,2.00$ and 3.00 (right).

In the following, we will discuss the silver ion density. The silver ions in the cell are located in a plane at $z=1 / 2$. In Figs. 3 and 4 , we show a Fourier synthesis of the observed scattering in the $a-b$-plane at $z=1 / 2$ for different temperatures.

The sulfur ions to the left and right of $\mathrm{Ag}$ are located $\Delta z \approx 0.19$ below the section whereas the pair above and below $\mathrm{Ag}$ lie above the section. Besides the expected maximum at the regular silver lattice site, we find silver density at positions $4 \mathrm{e}\left(\frac{1}{2} \frac{1}{2} \frac{1}{2}\right)$ and at 0.530 .250 .5 . The Fourier 

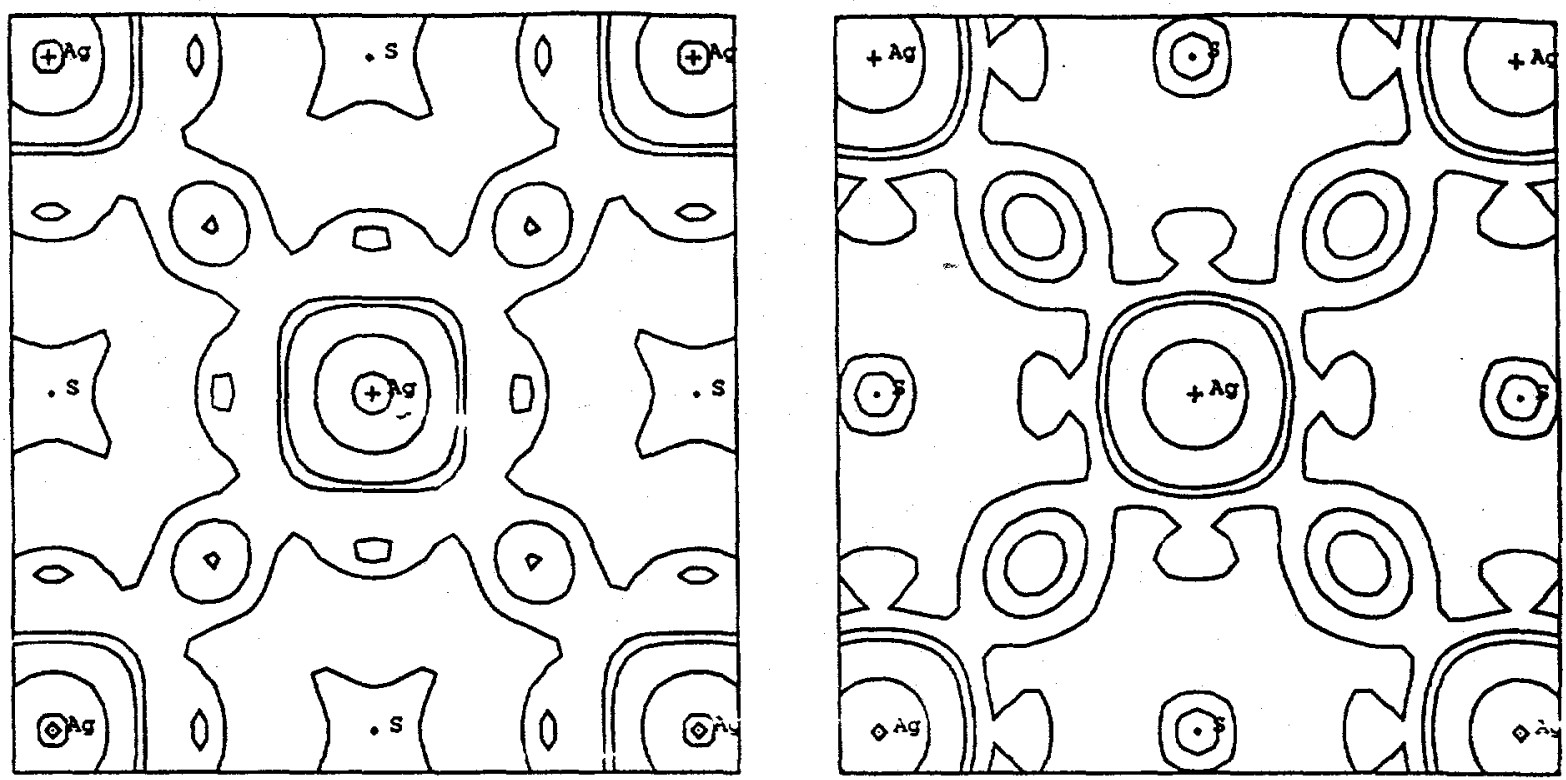

Figure 4: Fourier section $\left(F_{\text {obs }}\right)$ of $(\mathrm{LaO}) \mathrm{AgS}$ at $673 \mathrm{~K}$ (left) and $773 \mathrm{~K}$ (right) for $z=\frac{1}{2}$. The silver ions at the center of the diagram is located at position $3 \frac{1}{4} \frac{1}{4} \frac{1}{2}$. Contour values are $0.10,0.20,0.30$, $1.0,2.0$ (left) and $0.10,0.20,0.30$, and 1.00 (right).

plots at higher temperatures show, however, that position $4 \mathrm{e}$ is the more important one for the silver transport: there is continuous silver density from a regular $2 \mathrm{~b}$ via a $4 \mathrm{e}$ to a neighboring $2 \mathrm{~b}$ site. Obviously, the mobile silver ions move preferentially along $\langle 110\rangle$ via interstitial $4 \mathrm{e}$ sites, located at the mid point of the shared edge between neighboring $\mathrm{AgS}_{4}$ tetrahedra.

From the Fourier plots, we have extracted the silver scattering densities for the $2 \mathrm{~b}$ and $4 \mathrm{e}$ sites as well as the lowest value in between (saddle point) (Table 3 ).

\begin{tabular}{lllllll}
\hline T/K & 298 & 473 & 573 & 673 & 773 & mean \\
\hline \hline$\rho(2 b)$ & 4.98 & 3.03 & 2.05 & 2.22 & 1.73 & \\
$\rho(4 e)$ & 0.22 & 0.19 & 0.34 & 0.32 & 0.39 & \\
$\rho_{\text {saddle }}$ & 0.032 & 0.064 & 0.208 & 0.131 & 0.120 & \\
\hline$\Delta E(2 b, 4 e)$ & 0.062 & 0.085 & 0.054 & 0.072 & 0.053 & $0.065(13)$ \\
$\Delta E_{b}$ & 0.112 & 0.129 & 0.079 & 0.124 & 0.132 & $0.115(22)$ \\
\hline
\end{tabular}

Table 3: Fourier densities at silver sites $2 \mathrm{~b}$ and $4 \mathrm{e}$ and at the saddle point. Site energy difference and barrier height calculated using a Boltzmann ratio, cf. Eqs. (2) and (3).

If we consider the ratio of the population in the two types of sites to be proportional to a Boltzmann factor, we may calculate the energy difference between sites $2 \mathrm{~b}$ and $4 \mathrm{e}, \Delta E(2 b, 4 e)$ using

$$
\Delta E(2 b, 4 e)=k T \cdot \ln \left(\frac{\rho(2 b)}{2 \rho(4 e)}\right),
$$

where we have taken into account that there are twice as many $4 \mathrm{e}$ than $2 \mathrm{e}$ sites. Analogously, we calculate the barrier height, $\Delta E_{b}$ using

$$
\Delta E_{b}=k T \cdot \ln \left(\frac{\rho(2 b)}{2 \rho_{\text {saddle }}}\right)
$$

One might cross-check the obtained values given in Table 3 by plotting the population ratios versus inverse temperature (lin-log-plot) and extract the respective energies from the slopes. This proce- 
dure yields $(0.07 \pm 0.02) \mathrm{eV}$ and $(0.11 \pm 0.03) \mathrm{eV}$ for the site energy difference and barrier height, respectively. These results compare favourably with the average value of the energies calculated at single temperatures, cf. Table 3.

\section{CONCLUSION}

The fast silver ion conductor $(\mathrm{LaO}) \mathrm{AgS}$ was examined by temperature-dependent neutron diffraction and ac impedance measurements. Rietveld refinement of the powder data yields anisotropic temperature factors indicating enhanced silver mobility in the $a-b$-plane of the tetragonal structuro. Fourier density maps further support the view that ion conduction in $(\mathrm{LaO}) \mathrm{AgS}$ is performed in two dimensions. The preferred conduction path is via interstitial $\left(4 e, \frac{1}{2} \frac{1}{2} \frac{1}{2}\right)$ sites whose site energy is $0.07 \mathrm{eV}$ higher, compared to a regular Ag lattice site. The mean barrier for a silver ion hopping from $\mathrm{a} 2 \mathrm{~b}$ to a $4 \mathrm{e}$ site was found to be $0.11 \mathrm{eV}$, a value considerably lower than the activation energy determined from our conductivity measurements.

\section{ACKNOWLEDGEMENTS}

One of the authors (DW) is grateful for a fellowship granted by the Alexander von Humboldt Foundation. Travel support by the Ewald Wicke Stiftung is also gratefully acknowledged. We are indebted to Prof. Harry Tuller for assistance with the measurements of electrical conductivity. It is a pleasure to thank Dr. Kevin Eberman for his support during the neutron time-of-flight measurements and many fruitful discussions. We are indebted to Simine Short (ANL) for her assistance with the diffraction experiment. These measurements were performed at the Intense Pulsed Neutron Source at Argonne National Laboratory, a facility supported by the U.S. Department of Energy.

\section{REFERENCES}

1. M. Palazzi, C. Carcaly, J. Flahaut, J. Solid State Chem. 35, 150-155 (1980).

2. M. Palazzi, S. Jaulmes, Acta Cryst. B37, 1337-1339 (1981).

3. K. Ishikawa, S. Kinoshita, Y. Suzuki, S. Matsuura, T. Nakanishi, M. Aizawa, Y. Suzuki, J. Electrochem. Soc. 138, 1166-1170 (1991).

4. K. Ishikawa, A. Kumagai, O. Kohichi, S. Yamada, Y. Suzuki, Solid State Ionics 78, 41-48 (1995).

5. J.D. Jorgensen, J.Faber JR., J.M. Carpenter, R. K. Crawford, J. R. Haumann, R. L. Hittermann, R. Kleb, G. E. Ostrowski, F. J. Rotelle, T. G. Worlton, J. Appl. Cryst. 22, 321-333 (1989).

6. A. C. Larson, R. B. Von Dreele, GSAS General Structure Analysis System, Technical report, Los Alamos Neutron Scattering Center, 1994. 